\title{
Propiedad intelectual y emprendimientos tecnológicos. Casos de emprendimientos de la ciudad de Córdoba, Argentina
}

\author{
LORENA TALBOT-WRIGHT* \\ ANDRÉS COLOMBO**
}

\begin{abstract}
RESUMEN Los emprendedores tecnológicos son actores clave para dinamizar una economía. La base de su competencia en el mercado es la gestión del conocimiento tecnológico que desarrollan. Es en este punto donde cobran importancia los derechos de propiedad intelectual y su rol en la transferencia tecnológica. En este trabajo se estudiaron aspectos relativos a la gestión de la propiedad intelectual en emprendimientos tecnológicos de la ciudad de Córdoba, Argentina. Los resultados mostraron la importancia que la protección de intangibles tiene para los emprendedores, quienes la asocian a la competitividad de su emprendimiento principalmente en relación con la generación de barreras de entrada y la valorización de parte de los posibles socios. Asimismo, a pesar de que los emprendedores conocen la importancia de la protección de intangibles, carecen de comprensión sobre conceptos relacionados con la temática. La protección de los intangibles en emprendimientos de base tecnológica es un tema fundamental. Este trabajo sugiere que los emprendedores deberían considerar la gestión de los derechos de propiedad intelectual como una inversión y no un gasto para el desarrollo de los emprendimientos.
\end{abstract}

PALABRAS CLAVE propiedad intelectual, activos intangibles, emprendimientos, empresas de base tecnológica.
COMO CITAR ESTE ARTÍCULO

How to cite this article:

Talbot-Wright, L. y Colombo, A. (2019). Propiedad intelectual y emprendimientos tecnológicos. Casos de emprendimientos de la ciudad de Córdoba, Argentina. Revista Perspectiva Empresarial, 6(1), 129-136

Recibido: 24 de junio de 2018 Aprobado: 22 de octubre de 2018

\footnotetext{
* Doctoranda en Ciencias Económicas. Universidad Empresarial Siglo 21, Universidad Nacional de Córdoba, Córdoba, Argentina. E-mail: lorena.talbot@unc.edu.ar. ORCID: 0000-0002-1216-0103. Google Scholar: https://scholar.google.com/ citations?hl=es\&user=qefj7GgAAAJ.

** Doctor en Ciencias Químicas. Universidad Empresarial Siglo 21, Córdoba, Argentina. E-mail: andres.colombo@ues21.edu. ar. ORCID: 0000-0002-4097-8164. Google Scholar: https://scholar.google.com/citations?hl=es\&user=rG51KNAAAAAJ.
} 


\section{Intellectual property and technological entrepreneurships. Entrepreneurship cases in the city of Córdoba, Argentina}

ABSTRACT Technological entrepreneurs are key actors in invigorating an economy. The base of their competition in the market is the technological knowledge management they develop. It is at this point where intellectual property rights and their role in technological transfer become important. This work studied aspects related to intellectual property management in technological entrepreneurships in the city of Córdoba, Argentina. The results showed the importance intangibles protection has for entrepreneurs, who associate it with the competitiveness of their entrepreneurship, mainly regarding the generation of entry barriers and valuation by possible partners. Also, even though entrepreneurs know the importance of intangibles protection, they lack understanding of concepts related to this topic. Intangibles protection in technology-based entrepreneurships is a fundamental issue. This work suggests that entrepreneurs should consider intellectual property rights management as an investment and not as an expense for the development of entrepreneurships.

KEY WORDS Intellectual property, intangible assets, entrepreneurships, technology-based companies.

\section{Propriedade intelectual e empreendimentos tecnológicos. Casos de em- preendimentos da cidade de Córdoba, Argentina}

RESUMO Os empreendedores tecnológicos são atores chave para dinamizar uma economia. A base da sua competência no mercado é a gestão do conhecimento tecnológico que desenvolvem. É neste ponto onde cobram importância os direitos de propriedade intelectual e seu papel na transferência tecnológica. Neste trabalho se estudaram aspectos relativos à gestão da propriedade intelectual em empreendimentos tecnológicos da cidade de Córdoba, Argentina. Os resultados mostraram a importância que a proteção de intangíveis tem para os empreendedores, quem a associam à competitividade do seu empreendimento principalmente em relação com a geração de barreiras de entrada e a valorização de parte dos possíveis sócios. Assim mesmo, apesar de que os empreendedores conhecem a importância da proteção de intangíveis, carecem de compreensão sobre conceitos relacionados com a temática. A proteção dos intangíveis em empreendimentos de base tecnológica é um assunto fundamental. Este trabalho sugere que os empreendedores deveriam considerar a gestão dos direitos de propriedade intelectual como um investimento e não um gasto para o desenvolvimento dos empreendimentos.

PALAVRAS-CHAVE propriedade intelectual, ativos intangíveis, empreendimentos, empresas de base tecnológica. 


\section{Introducción}

Si bien existen muchas definiciones, desde la perspectiva empresarial puede decirse que los emprendedores son individuos que transforman ideas en organizaciones rentables (Lederman et al., 2014). Los emprendedores tecnológicos buscan crear empresas de base tecnológica -EBT(conocidas también como empresas dinámicas o empresas tecnológicas sin más). Este tipo de emprendimientos son un motor del desarrollo económico de una región y generan beneficios directos como la creación de nuevos empleos y la diversificación de la matriz productiva; no obstante, la capacidad de generación de las EBT -de una región o de un país- depende de diversos factores.

En los últimos años, los países latinoamericanos han demostrado algunas condiciones favorables para el desarrollo de emprendimientos innovadores tales como el aumento de la demanda y el consiguiente incremento de las oportunidades de negocio que contribuyen a una mayor actividad emprendedora. Pese a esto, aún existen algunas deficiencias que impiden un aumento significativo en el número de EBT. Entre estas, se puede encontrar el escaso capital humano emprendedor; una escasa masa crítica de emprendedores con capacidades y vocaciones para emprender, algo muy común no solo en Argentina sino en el resto de los países de América Latina. Por otro lado, si bien el primer decenio de este siglo ha mostrado un mercado latinoamericano creciente y dinámico esta tendencia se ha resentido en los últimos años; impactando de manera negativa en los ecosistemas emprendedores de los países. A su vez, dos aspectos se vuelven relevantes cuando se habla de emprendimientos tecnológicos latinoamericanos: (i) el muy bajo aporte de la ciencia y la tecnología para el desarrollo de innovaciones y (ii) el acceso al financiamiento. En este último caso, aunque se viene trabajando en la disponibilidad de fondos públicos para emprendedores y en la promoción de la inversión privada en emprendimientos, la cadena de financiamiento para Latinoamérica sigue mostrando muchas debilidades. Particularmente es necesario que se trabajen los mecanismos de capital semilla, desarrollo, expansión y aceleración de EBT no solo aumentando los fondos disponibles sino articulando esta cadena de financiamiento. A pesar de estos aspectos, desde una perspectiva de largo plazo, resulta claro que la región ha avanzado en el desarrollo del emprendedorismo y en la conformación de ecosistemas emprendedores capaces de nuclear actores que promuevan la actividad (como, por ejemplo, incubadoras, aceleradoras, organismos de financiamiento, empresas, inversores entre otros).

Los EBT se caracterizan por conocimientos (know-how) en los que se basan sus características innovadoras y su competencia en un mercado potencial. En muchos casos este tipo de emprendimientos surge a partir de trabajos de grado, posgrado o trabajos de investigación en el seno de las universidades, por lo que representan una vía muy importante para la transferencia de estos resultados. Así, la universidad puede darle valor a sus conocimientos a través de la creación de empresas; específicamente a través de spin-off académicos o EBT (García Mandaloniz, 2013). Aunque muchas veces no es posible determinar de manera fehaciente la contribución que estas EBT hacen al sector socioproductivo, sí resulta claro que los esfuerzos para promover este tipo de empresas apuntan a la transformación de la inversión en ciencia básica en crecimiento económico, nuevos empleos y el aumento de la competitividad (Drucker, 1985). En este punto cobran importancia los derechos de propiedad intelectual -DPI-, definidos como "los derechos exclusivos otorgados por el Estado sobre las creaciones del intelecto humano, en particular, las invenciones, las obras literarias y artísticas, y los signos y diseños distintivos utilizados en el comercio" (OMPI, 2017). La propiedad intelectual -PI- se divide en dos categorías principales: los derechos de propiedad industrial (patentes, modelos de utilidad, marcas, diseños industriales, secretos comerciales, obtenciones vegetales e indicaciones geográficas) y el derecho de autor y derechos conexos (obras literarias y artísticas) (OMPI, 2017).

La ciudad de Córdoba es una de las regiones más importantes de Argentina en cuanto al fomento del emprendedorismo tecnológico. Particularmente cuenta con una gama muy amplia de instituciones de apoyo al emprendedor tales como incubadoras, aceleradoras, agencias estatales y diversas instituciones. A pesar de la trayectoria de la región en el desarrollo del emprendedorismo científico y tecnológico, no está clara la relación que los emprendedores tecnológicos tienen con la gestión de los activos intangibles 
tanto en lo relativo a su percepción como elemento de valor para su propio proyecto como a las distintas actividades y herramientas que existen para poder manejar de manera eficiente este tipo de activos en el seno de una EBT. En este trabajo se propone analizar aspectos relativos a la PI en EBT de Córdoba, diagnosticando la situación de estos emprendimientos en relación con los activos intangibles y proponiendo herramientas para su análisis.

\section{Metodología aplicada}

En este trabajo se analizaron EBT de la ciudad de Córdoba, Argentina. En todos los casos, y en mayor o menor medida, estos proyectos buscan transformarse en nuevas EBT y su característica común es que basan su actividad en las aplicaciones de nuevos descubrimientos científicos o tecnológicos para la generación de nuevos productos, procesos o servicios. El Ecosistema Emprendedor de Córdoba -EECBA- es una asociación que nuclea a las instituciones de la región que trabajan en el fomento del emprendedorismo. Según el último informe del EECBA (2017), en el primer semestre de 2017 se incubaron y/o aceleraron en la ciudad de Córdoba 56 EBT. En este texto, se trabajó sobre una muestra de 40 emprendimientos tecnológicos.

El método de recogida de la información comprendió la realización de envíos por correo electrónico y el análisis de la información colectada por parte del grupo de investigación (OCDE, 2006). Se diseñó un cuestionario individual por empresa para indagar sobre la importancia de la PI en este tipo de emprendimientos. Las respuestas se registraron a través de un formulario en línea que permitió de manera simple sistematizarlas. Se enviaron las encuestas a la base de datos completa (40 empresas) y luego se reenvió a los encuestados que no habían respondido, obteniéndose 36 respuestas (esto representa el $90 \%$ de las respuestas).

Se trabajaron tres objetivos en la concepción del formulario: (i) generar un formulario con información adaptada al lenguaje del encuestado; (ii) trabajar en la simpleza, minimizando el tedio y la fatiga y (iii) adaptar las preguntas de forma que sean claras para evitar confusión (Malhotra, 1997). La selección de las preguntas se orientó a contribuir con el objetivo de la investigación; se descartaron aquellas que no aportaron a la discusión.

El cuestionario se trabajó partiendo de preguntas generales a preguntas específicas, introduciendo al encuestado en la temática. Se enfocó en que la mayoría de las preguntas fueran estructuradas tipo dicotómicas. En aquellos temas donde se quería ahondar se introdujeron preguntas abiertas como, por ejemplo, en la pregunta 5: “ipor qué cree importante, proteger o no sus activos intangibles?" Este tipo de pregunta exploratoria nos permitió comprender la importancia de la temática en relación al estadio de la empresa analizada.

\section{Resultados y discusión}

Se analizó el conocimiento de los emprendimientos con respecto a elementos relativos al sistema de PI. De los encuestados, el 91,6 \% aseguró que su emprendimiento se basa en un activo intangible; conceptualizando como activo intangible al capital humano y los conocimientos técnicos, invenciones, marcas, dibujos y modelos, así como otros frutos intangibles de la capacidad creadora e innovadora del emprendimiento. En el corazón de la actividad de una EBT reside la gestión de su know-how. Es así que el capital intangible se asocia claramente con innovación tecnológica y una mejora en la competitividad: aquellas empresas que brindan productos y servicios relacionados con la tecnología (tal como es el caso de este estudio) son intrínsecamente innovadoras, facilitando y derramando innovación en otros sectores económicos (Díaz Chao y Torrent Sellens, 2010; Parga Dans, 2011). En este sentido la viabilidad y éxito de una empresa va de la mano de los recursos intangibles que esta pueda movilizar (Itami and Roehl, 1987).

Si bien es clara la importancia que tienen los activos intangibles para el crecimiento y mejor desempeño de una empresa, la cantidad de trabajos que analizan el impacto de este tipo de activos en emprendimientos es menor (Mann and Sager, 2007). En esta línea, Presutti et al. (2007) sugieren que el conocimiento y el capital social 
son activos intangibles que permiten acelerar el crecimiento internacional de un emprendimiento. Asimismo, en un estudio sobre emprendimientos en el Reino Unido, Helmers y Rogers (2011) encontraron que la tasa de crecimiento anual de los emprendimientos que patentan es entre el 8 $\%$ y el $27 \%$ respecto de aquellos que no lo hacen; sugiriendo también que la decisión de proteger los activos intangibles aumenta la tasa de supervivencia de un emprendimiento en sus primeros cinco años de vida. Por otro lado, Parga Dans et al. (2013) hacen un aporte al estudio de la innovación organizacional en empresas de servicios intensivos en conocimiento donde los activos intangibles resultan clave como fuente de valor. A pesar de esto, en este tipo de empresas, las innovaciones son difíciles de cuantificar y el sistema de patentes no se adecúa a las particularidades de un sector donde las innovaciones tecnológicas no son las más importantes (Parga Dans, 2011).

Para Ulrich (2001), el único bien revalorizable de una empresa es el capital humano dado que los bienes materiales van perdiendo valor con el tiempo. En esta línea, el 39,53 \% de los emprendedores tecnológicos encuestados indicó que su emprendimiento se basa en conocimientos específicos que posee el equipo emprendedor. Este intangible posee un valor fundamental en la gestión de la innovación puesto que el conocimiento es indisociable de la persona que lo posee y por lo tanto, en este tipo de emprendimientos, la capacidad global de un equipo para encontrar soluciones es mayor que las capacidades individuales de los miembros que la componen (Benavides y Quintana, 2003).

El 32,52 \% de los encuestados aseguró que sus empresas se basan en una invención, diferenciándola de los conocimientos específicos del capital humano $(39,53 \%)$ dado que consideran que han materializado la idea y los conocimientos en un producto o procedimiento innovador. Asimismo, el 13,95 \% de los emprendedores tiene como activo intangible el software; solo el 9,30 $\%$ protegió su marca y el 4,65 \% presenta dibujos o modelos industriales. El perfil obtenido condice con el tipo de emprendimientos encuestados, ya que estos buscan desarrollarse como EBT en las que por su carácter dinámico e innovador el conocimiento embebido se transforma en un pilar clave de su crecimiento y desarrollo. Aquí, es importante conocer el origen de este conocimiento para determinar la titularidad del mismo de acuerdo a lo que estipula la legislación argentina (Ley № 24.481, “Ley de patentes de invención y modelos de utilidad").

El 51,45 \% de los proyectos encuestados en este trabajo tiene relación con alguna universidad de la región. El tipo de relación es vinculante con la titularidad del derecho de PI. Por ejemplo, el régimen de propiedad intelectual de la Universidad Nacional de Córdoba -UNC- (una de las universidades más importantes de la región) distingue entre: un autor que percibe salario o beca o utiliza infraestructura, insumos o equipamiento de la universidad para generar un desarrollo, de aquel que solamente es estudiante y no percibe retribuciones de su trabajo ${ }^{1}$. En relación con el grupo de emprendedores encuestados con vínculos con la universidad, un 23,81 \% no tienen vinculación con la UNC debido a que son egresados o estudiantes. El 76,19 \% restante sí presenta relación con la UNC, ya que son doctorandos o docentes/ investigadores de la universidad. En este punto se produce el primer dilema que concierne a la gestión de la PI en estos emprendimientos: ¿cómo se generó ese activo? ¿Con qué insumos? ¿Se debería transferir al nuevo emprendimiento? Es claro que en estos casos los investigadores universitarios y a la vez emprendedores están presentes en las dos partes interesadas en los derechos de PI (universidad y emprendimiento privado), por lo que desde la universidad se deben plantear alternativas para hacer eficiente esta transferencia de conocimientos. En este sentido, de los encuestados, el 37,5 \% indicó que su desarrollo se generó sin vinculación con la UNC; mientras que el resto (62,6 \%), sí desarrollaron el componente innovador de su emprendimiento en relación con la institución. Para el caso de los emprendedores

1 Artículo 3, inciso 1, del Régimen de Propiedad Intelectual de la UNC: “la Propiedad Intelectual pertenecerá a la UNC, salvo pacto en contrario, siempre que las creaciones sean obtenidas por los autores durante el curso de una actividad en la UNC, que tenga por objeto total o parcial la realización de actividades de creación intelectual y que hubiesen sido realizadas con aportes de la UNC a través del pago de salarios, becas, contratos, relación laboral o de servicios y/o con apoyo de subsidios de la UNC o actividades de intercambio, o mediante el uso de bienes o medios proporcionados por la UNC". 
que son también investigadores, existe una particularidad en relación a su pertenencia a la carrera de investigador y/o las actividades como becario en el sistema de ciencia y tecnología de Argentina: la figura de doble dependencia. Esta figura implica que un investigador perciba retribuciones económicas de dos instituciones: del Consejo Nacional de Investigaciones Científicas y Técnicas -CONICET-, responsable en muchos casos del salario por el desarrollo de tareas de investigación; de las universidades, encargadas de pagar el salario de un investigador en su función como docente (y según el tipo de cargo, también por actividades de investigación) y brindar infraestructura y equipamiento para el desarrollo de la actividad científica. Por esta razón, un investigador de doble dependencia (CONICETuniversidad) tiene dos empleadores a los cuales debe reconocer como titulares de las invenciones realizadas en el ámbito de esta relación laboral. El $62,5 \%$ de los emprendedores encuestados indicó que utilizan como corazón de su emprendimiento invenciones cuya titular es la UNC y en el 16,7 \% también el CONICET. Así, resulta claro que es necesario clarificar el origen de estas invenciones en casos como los descritos donde la doble relación de dependencia impacta en los DPI.

Los resultados obtenidos mostraron que la mayoría de los emprendimientos $(90,2 \%)$ conoce qué es la PI, así como el 83,3 \% de los emprendedores mostró interés por proteger sus activos intangibles. Entre los comentarios analizados se destaca que la gestión de la PI, les da una ventaja competitiva al obtener el derecho exclusivo de su invención y de esta manera protegerse ante copias de futuros competidores. En ese mismo orden de ideas, el 58,3\% de los emprendedores encuestados indican que han realizado consultas relacionadas con PI tanto en la UNC como en estudios privados. A su vez, el 41,7 \% de los emprendedores indicaron que proteger sus activos es importante a la hora de negociar con potenciales inversores dado que estos ponderan de manera positiva aquellos emprendimientos que tengan estos intangibles preservados. Esta es otra de las ventajas que el sistema de protección de derechos de PI brinda a los emprendimientos tecnológicos; esto es, la posibilidad de proteger sus desarrollos para que inversores potenciales conozcan las posibilidades que tiene la invención en el mercado frente a los competidores. El 24,7 \% de los encuestados indicó que al menos un inversor o cliente le consultó si los desarrollos estaban protegidos por DPI. En relación con este tipo de estrategias el foco se centra en si la innovación genera al emprendimiento una ventaja competitiva, disminuyendo los riesgos y maximizando los rendimientos, fruto del uso exclusivo que le brinda el DPI.

De manera antagónica, de este estudio surgen emprendimientos $(16,7 \%)$ que no mostraron interés por proteger sus activos ya sea porque están en una etapa incipiente del desarrollo de su EBT o porque piensan que el sistema de protección es lento. En este último caso, algunos emprendedores comentaron que el desarrollo tecnológico avanza rápidamente y al momento de la protección ese conocimiento ya es obsoleto para el mercado.

Los DPI no deben ser vistos solamente como un mecanismo legal de protección: desde que sean bien utilizados, son fuente de información que permite conocer los desarrollos e investigaciones en el mundo; y a través de estos, es posible interiorizarlos en los campos donde se desea trabajar. Una herramienta muy interesante para indagar rápidamente sobre el estado del arte asociado con la tecnología que maneja un emprendimiento es la vigilancia tecnológica -VT-. La VT se ocupa de las tecnologías disponibles o que acaban de aparecer -capaces de intervenir en nuevos productos o procesos, avances científicos y técnicos- fruto de la investigación básica y aplicada, los materiales y su cadena de transformación (Ministerio de Ciencia, Tecnología e Innovación Productiva, 2015). Consultados sobre la VT, el $100 \%$ de los emprendedores encuestados se interesó en conocer aspectos relacionados con la VT y su emprendimiento. Este resultado es contundente. La Guía Nacional de Vigilancia e Inteligencia Estratégica [VeIE] (Ministerio de Ciencia, Tecnología e Innovación Productiva, 2015) sugiere que la frecuencia de emisión, cantidad de novedades y/o tipo de información debe ser definida según la necesidad de cada cliente. 


\section{Conclusiones}

En este trabajo se estudiaron aspectos relativos a la gestión de la PI en EBT de Córdoba, Argentina. De lo analizado, resulta claro que los emprendedores conocen la importancia de la protección de intangibles; no obstante, no es evidente que manejen conceptos y aspectos específicos relacionados con la temática. Esta última observación se refleja en la heterogeneidad de respuestas y en el desconocimiento de la legislación vigente (tal como quedó reflejado en algunas respuestas) que ponen de manifiesto la falta de información relacionada con aspectos clave como la titularidad de los DPI y la legislación aplicable en cada caso. Es posible que esto se deba a la carencia de espacios de formación en el área. En esta línea, Pitkethly (2011) indicó que existe una falta sustancial de comprensión del sistema de PI entre las pequeñas empresas del Reino Unido. Más allá de esto, los emprendedores tecnológicos son conscientes de la importancia que tienen los DPI para el éxito de su emprendimiento; algo que está asociado a la generación de barreras de entrada. No obstante, es evidente la necesidad de estudios posteriores que analicen el impacto de la propiedad intelectual en la supervivencia y desarrollo de emprendimientos haciendo hincapié en el proceso de internacionalización de una empresa y la relación con sus activos intangibles.

La protección de los intangibles en EBT es un tema de gran interés, tanto para los emprendedores como para las universidades e instituciones relacionadas. Lo analizado sugiere que la gestión de los DPI debería considerarse una inversión y no un gasto en el desarrollo de los emprendimientos, con más énfasis en la instancia donde se genera el I+D+i. Así, emprendedores y EBT deberían analizar la posibilidad de incluir en sus presupuestos la VT acompañada de informes del estado de desarrollo como herramientas indispensables. Esta información permitiría realizar evaluaciones periódicas sobre los avances tecnológicos donde están inmersos sus emprendimientos y por lo tanto mejorar la toma de decisiones. Por otro lado, sería de suma importancia generar espacios de capacitación específica relacionadas a los DPI; haciendo foco en el valor de las protecciones, en las ventajas y desventajas y aportando a la discusión fundamental de reglamentación y leyes. Adicionalmente las universidades deberían ahondar en procesos y capacidades propias para gestionar y ayudar a ser eficientes en el desarrollo de DPI en los emprendimientos.

\section{Referencias}

Benavides, C. y Quintana, C. (2003). Gestión del conocimiento y calidad total. Madrid, España: Ediciones Diaz de Santos S.A.

Díaz Chao, A. y Torrent Sellens, J. (2010). ¿Pueden el uso de las TIC y los activos intangibles mejorar la competitividad? Un análisis empírico para la empresa catalana. Estudios de Economía Aplicada, 28 (2), 1-24.

Drucker, P.F. (1985). Innovation and Entrepreneurship: Practice and Principles. New York, USA: Harper \& Row.

EECBA. (2017). Ecosistema Emprendedor de Córdoba. Recuperado de http://eecordoba.org/.

García Mandaloniz, M. (2013). Los viveros de empresas para fortalecer la transferencia de la tecnología y el conocimiento desde la Universidad a la Sociedad: el reto de la Universidad emprendedora. Revista Pensar en Derecho, 3, 141-183.

Helmers, H. and Roger, M. (2011). Does patenting help high-tech start-ups? Research Policy, 40 (7), 10161027.

Itami, H. and Roehl, T.W. (1987). Mobilizing Invisible Assets. Cambridge, USA: Harvard University Press.

Lederman, D. et al. (2014). El emprendimiento en América Latina: muchas empresas y poca innovación. Washington, USA: Banco Mundial.

Malhotra, N. (1997). Investigación de mercados. Un enfoque práctico. Ciudad de México, México: Prentice Hall Hispanoamericana S.A.

Mann, R. and Sager, T.W. (2007). Patents, venture capital, and software start-ups. Research Policy, 36 (2), 193-208.

Ministerio de Ciencia, Tecnología e Innovación Productiva. (2015). Guía Nacional de Vigilancia e Inteligencia Estratégica [VeIE]. Buenas prácticas para generar sistemas territoriales de gestión VeIE. 
Buenos Aires, Argentina: Ministerio de Ciencia, Tecnología e Innovación Productiva.

OCDE. (2006). Manual de Oslo de la OCDE. Guía para la recogida e interpretación de datos sobre innovación. Madrid, España: Grupo Tragsa.

OMPI. (2017). Organización Mundial de la Propiedad Intelectual. Recuperado de http://www.wipo.int.

Parga Dans, E. (2011). Innovación y emergencia de un servicio intensivo en conocimiento: el caso de la arqueología comercial. Santiago de Compostela, España: Universidad de Santiago de Compostela.

Parga Dans, E. et al. (2013). La innovación organizativa y de gestión como motor de dinamización empresarial. Journal of Technology Management \& Innovation, 8 (2), 132-143.

Pitkethly, R. (2011). UK Intellectual Property Awareness Survey 2010. Report for the UK Intellectual Property Office. Oxford, United Kingdom: St. Peter's College, Oxford Intellectual Property.

Presutti, M. et al. (2007). Knowledge acquisition and the foreign development of high-tech start-ups: A social capital approach. International Business Review, 16 (1), 23-46.

Ullrich, H. (2001). Intellectual Property, Access to Information and Antitrust: Harmony, Disharmony and International Harmonization. En Dreyfuss, R.C., Zimmerman, D. L. and First, H. (Ed.), Expanding the boundaries of intellectual property-innovation policy for the knowledge society (pp. 365-402). Oxford, United Kingdom: Oxford University Press. 